\title{
Optimal Therapy for Stroke Prevention in Atrial Fibrillation: Is it Left Atrial Appendage Closure?
}

\author{
Harold Rivner ${ }^{1}$ and Jeffrey Goldberger ${ }^{1}$ \\ ${ }^{1}$ University of Miami
}

January 28, 2022

Optimal Therapy for Stroke Prevention in Atrial Fibrillation: Is it Left Atrial Appendage Closure?

Harold Rivner, $\mathrm{MD}^{1}$

Jeffrey J. Goldberger, MD, MBA ${ }^{1}$

1 Cardiovascular Division, Department of Medicine, University of Miami Leonard M. Miller School of Medicine, Miami, FL

\section{Corresponding author:}

Jeffrey J. Goldberger, M.D.

University of Miami Miller School of Medicine

Cardiovascular Division

$1120 \mathrm{NW} 14^{\text {th }}$ St.

Miami, FL 33136

Telephone: (305) 243-8092

Fax: (305) 243-1731

E-mail:j-goldberger@miami.edu

Sarris-Michopoulos et al. reviewed the role of the left atrial appendage (LAA) in atrial fibrillation (AF) related stroke and current techniques for targeting it for stroke reduction. ${ }^{1}$ The authors propose that further trials to assess LAA exclusion as an alternative to anticoagulation for patients with AF should strongly be considered. Any such consideration should take into account the potential risks and benefits, and ultimately be supported by randomized clinical trial data. The latter is lacking at the present time, so we will consider the potential risks and benefits.

The LAA is often considered to be a vestigial portion of the left atrium; however, it does have a physiologic role in humans. The LAA is a remnant of the embryonic left atrium that develops during the third week of fetal life. ${ }^{2}$ It has several functions that continue into adulthood including serving as a reservoir during left ventricular systole, a conduit for blood from the pulmonary veins into the left ventricle during diastole, a chamber to augment left ventricular filling in late diastole, and a filling chamber for volume reserve. ${ }^{2}$ Furthermore, the LAA plays an important endocrine role. It contains stretch receptors to excrete atrial natriuretic peptide in response to changes in left atrial pressure to increase natriuresis. ${ }^{2}$ Removal of the LAA eliminates these functional roles. Interestingly, it has been shown that patients undergoing LAA exclusion have elevated left atrial pressure. ${ }^{3}$ 
It has been established that patients with AF undergoing cardiac surgery for another indication benefit from LAA occlusion at the time of surgery. In the LAAOS III (Left Atrial Appendage Occlusion during Cardiac Surgery to Prevent Stroke) multicenter trial, 4,811 participants with a mean $\mathrm{CHA}_{2} \mathrm{DS}_{2}$-VASc score of 4.2 were randomized to undergo either LAA occlusion or no occlusion. The patients were followed for a mean of 3.8 years. Stroke or systemic embolism occurred in 114 participants $(4.8 \%)$ in the occlusion group and $168(7.0 \%)$ in the no-occlusion group (HR 0.67, 95\% CI: $0.53-0.85, \mathrm{p}=0.001) .{ }^{4}$ Of note, $76.8 \%$ of patients remained on oral anticoagulation at 3 years, supporting the role of LAA occlusion as an adjunct to and not a replacement for anticoagulation.

The concept of LAA occlusion/ligation as an alternative to anticoagulation is considered based on the data that approximately $90 \%$ of non-rheumatic atrial fibrillation left atrial thrombi can be localized to the LAA. ${ }^{5}$ If correct, this implies that about $10 \%$ of left atrial thrombi in patients with atrial fibrillation would not be addressed by LAA occlusion. If anticoagulation is not contraindicated, there would therefore theoretically still be benefit for anticoagulation to address these non-LAA thrombi. Future clinical trials to assess the additional benefit for anticoagulation in patients who have undergone LAA occlusion/ligation would be important to address this question but will be challenging due to the noninferiority design that would be required and the low event rate.

Available data from trials comparing LAA closure to warfarin have provided some evidence of lower efficacy for LAA closure in reducing ischemic stroke. The most studied device, the endovascularly placed Watchman, shows a trend towards reduced efficacy in preventing ischemic stroke. The five-year outcomes of the PREVAIL (Evaluation of the WATCHMAN LAA Closure Device in Patients with Atrial Fibrillation Versus Long Term Warfarin Therapy) did not meet noninferiority for the first composite coprimary endpoint of stroke, systemic embolism, or cardiovascular/unexplained death. A meta-analysis of the PREVAIL and PROTECT AF (WATCHMAN Left Atrial Appendage system for embolic Protection in Patients with Atrial Fibrillation) trials did show a similar composite endpoint of stroke, systemic embolism, or cardiovascular death between groups (HR 0.82, 95\% CI 0.58-1.17 p = 0.27). However, there was a numerically higher rate of ischemic stroke in the Watchman group (HR 1.71, 95\% CI 0.94-3.11, $\mathrm{p}=0.08$ ) but a lower rate of hemorrhagic stroke (HR 0.20, 95\% CI 0.07-0.56, $\mathrm{p}=0.0022){ }^{6}$

It is debatable how to apply these data in the current age of non-warfarin direct oral anticoagulant (DOAC) use. Compared to warfarin, DOACs have a lower risk of both stroke/systemic embolism (HR 0.81, 95\% CI 0.74-0.89) and intracranial bleeding (HR 0.45, 95\% CI 0.37-0.56). ${ }^{7}$ The PRAGUE-17 (Left Atrial Appendage closure versus Novel Anticoagulation Agents in Atrial Fibrillation) trial compared Watchman closure with DOACs. Closure with the Watchman device met non-inferiority for the composite endpoint of cardiovascular death, all-stroke/transient ischemic attack, clinically relevant bleeding, and device/procedure-related complications) ( $\mathrm{sHR}=0.81,0.56-1.18, \mathrm{p}=0.27$, $\mathrm{p}$-value for noninferiority $=0.006)$. There was a numerical but not statistically significant increase in the all-stroke rate (HR 1.38, 0.63-3.03, $\mathrm{p}=0.42){ }^{8}$

It is unclear how the data from Watchman would extend to surgical LAA occlusion. While these surgical techniques appear promising, the data presented in the review article by Sarris-Michopoulos et al. is entirely from observational studies. ${ }^{1}$ The total number of subjects in these studies is 442 patients. ${ }^{9-11}$ This is less than aforementioned randomized Watchman trials which enrolled 707 patients in the PROTECT AF, 407 patients in PREVAIL, and 402 patients in PRAGUE-17. Even these studies enrolled many fewer patients than the seminal trials of DOACs versus warfarin: 18,133 in RE-LY (Randomized Evaluation of Long-Term Anticoagulation Therapy) ${ }^{12}$, 14,264 in ROCKET-AF (Rivoxaraban Once Daily Oral Direct Factor XA Inhibition Compared with Vitamin K Antagonism for Prevention of Stroke and Embolism Trial in Atrial Fibrillation) ${ }^{13}$, 18,201 patients in ARISTOTLE (Apixaban for Reduction in Stroke and Other Thromboembolic Events in Atrial Fibrillation) ${ }^{14}$, and 21,105 patients in ENGAGE AF-TIMI 48 (The Effective Anticoagulation with a Factor Xa Next Generation in Atrial Fibrillation-Thrombolysis in Myocardial Infarction 48) ${ }^{15}$ for a total of 71,703 patients. It is therefore difficult to make conclusions regarding the safety and efficacy of these LAA occlusion techniques. Furthermore, we must be careful not to extend the findings of one LAA occlusion method to another. 
We agree with Sarris-Michopoulos et al. that this is an exciting field that needs further randomized trials. The technology behind nonsurgical LAA occlusion techniques such as Watchman, Amplatzer Amulet, and Lariat will likely continue to evolve. There is good reason to hope that new iterations of these devices will have less complication rates but whether they can become the primary approach to prevent ischemic strokes is unknown. For example, the Watchman FLX device has an improved design to increase safety and efficacy. We look forward to the results of the CHAMPION-AF study that will randomize patients to Watchman FLX versus DOAC treatment. ${ }^{16}$ Similar efforts are needed to provide evidence from randomized controlled trials for surgical techniques such as LAA clipping. While the therapeutic landscape is being investigated, it is also important to point out that there is tremendous opportunity to refine our diagnostic approach to stroke prevention in patients with AF. The current standard assessment - the $\mathrm{CHA}_{2} \mathrm{DS}_{2}-\mathrm{VASc}$ score - has only a mediocre C-statistic estimated to range from $0.61-0.68 .{ }^{17,18}$ The cumulative one year risk for thromboembolic events in patients with atrial fibrillation with $\mathrm{CHA}_{2} \mathrm{DS}_{2}$-VASc scores [?] 2 (accounting for $89 \%$ of the AF patients) who are not anticoagulated is estimated at $4.5 \% \cdot{ }^{19}$ This indicates that the majority of patients with atrial fibrillation do not experience stroke but are subjected to the risks of anticoagulation. Better diagnostic approaches to identify which patients are at risk and which are not could have tremendous public health ramifications. There is active current interest in defining the atrial myopathy or cardiopathy that is associated with stroke. ${ }^{20-22}$ Several techniques have been under investigation, including LAA size/morphology ${ }^{23,24}$ electrocardiogram P wave morphology, ${ }^{25,26}$ echocardiographic LA strain, ${ }^{27,28}$ epicardial adipose tissue ${ }^{29}$ troponin and $\mathrm{N}$ terminal pro-brain natriuretic peptide levels ${ }^{30,31}$, and $4 \mathrm{D}$ flow MRI to evaluate left atrial and LAA stasis. ${ }^{32-34}$ There is also ongoing research into "pill-in-pocket" anticoagulation that would use continuous smartwatch monitoring to target anticoagulation to intermediate risk patients during high risk time periods. ${ }^{35}$ Combined efforts in the diagnostic and therapeutic realm are most likely to achieve improved clinical results for stroke prevention in atrial fibrillation.

\section{References}

1. Sarris-Michopoulos P, Magarakis M. Left atrial appendage exclusion in patients with atrial fibrillation: should it become standard of care? J Card Surg.

2. Regazzoli D, Ancona F, Trevisi N, et al. Left atrial appendage: physiology, pathology, and role as a therapeutic target. Biomed Res Int. 2015;2015:205013. doi:10.1155/2015/205013

3. Tabata T, Oki T, Yamada H, et al. Role of left atrial appendage in left atrial reservoir function as evaluated by left atrial appendage clamping during cardiac surgery.Am J Cardiol. 1998;81(3):327-332. doi:10.1016/s0002-9149(97)00903-x

4. Whitlock RP, Belley-Cote EP, Paparella D, et al. Left atrial appendage occlusion during cardiac surgery to prevent stroke. N Engl J Med. 2021;384(22):2081-2091. doi:10.1056/NEJMoa2101897

5. Blackshear JL, Odell JA. Appendage obliteration to reduce stroke in cardiac surgical patients with atrial fibrillation. Ann Thorac Surg. 1996;61(2):755-759. doi:10.1016/0003-4975(95)00887-X

6. Reddy Vivek Y., Doshi Shephal K., Kar Saibal, et al. 5-Year outcomes after left atrial appendage closure. J Am Coll Cardiol. 2017;70(24):2964-2975. doi:10.1016/j.jacc.2017.10.021

7. Carnicelli AP, Hong H, Connolly SJ, et al. Direct oral anticoagulants versus warfarin in patients with atrial fibrillation: patient-level network meta-analyses of randomized clinical trials with interaction testing by age and sex.Circulation. Published online January 5, 2022. doi:10.1161/CIRCULATIONAHA.121.056355

8. Osmancik P, Herman D, Neuzil P, et al. 4-Year outcomes after left atrial appendage closure versus nonwarfarin oral anticoagulation for atrial fibrillation. $J$ Am Coll Cardiol. 2022;79(1):1-14. doi:10.1016/j.jacc.2021.10.023

9. van Laar C, Verberkmoes NJ, van Es HW, et al. Thoracoscopic left atrial appendage clipping: a multicenter cohort analysis. JACC Clin Electrophysiol. 2018;4(7):893-901. doi:10.1016/j.jacep.2018.03.009 
10. Branzoli S, Marini M, Guarracini F, et al. Epicardial standalone left atrial appendage clipping for prevention of ischemic stroke in patients with atrial fibrillation contraindicated for oral anticoagulation. $J$ Cardiovasc Electrophysiol. 2020;31(8):2187-2191. doi:10.1111/jce.14599

11. Cartledge R, Suwalski G, Witkowska A, et al. Standalone epicardial left atrial appendage exclusion for thromboembolism prevention in atrial fibrillation.Interact Cardiovasc Thorac Surg. Published online December 6, 2021. doi:10.1093/icvts/ivab334

12. Connolly SJ, Ezekowitz MD, Yusuf S, et al. Dabigatran versus warfarin in patients with atrial fibrillation. N Engl J Med. 2009;361(12):1139-1151. doi:10.1056/NEJMoa0905561

13. Patel MR, Mahaffey KW, Garg J, et al. Rivaroxaban versus warfarin in nonvalvular atrial fibrillation. N Engl J Med. 2011;365(10):883-891. doi:10.1056/NEJMoa1009638

14. Granger CB, Alexander JH, McMurray JJV, et al. Apixaban versus warfarin in patients with atrial fibrillation. $N$ Engl J Med. 2011;365(11):981-992. doi:10.1056/NEJMoa1107039

15. Giugliano RP, Ruff CT, Braunwald E, et al. Edoxaban versus warfarin in patients with atrial fibrillation. $N$ Engl J Med. 2013;369(22):2093-2104. doi:10.1056/NEJMoa1310907

16. CHAMPION-AF clinical trial - full text view - clinicaltrials.gov. Accessed January 18, 2022. https://clinicaltrials.gov/ct2/show/NCT04394546

17. Lip GYH, Nieuwlaat R, Pisters R, Lane DA, Crijns HJGM. Refining clinical risk stratification for predicting stroke and thromboembolism in atrial fibrillation using a novel risk factor-based approach: The Euro Heart Survey on atrial fibrillation. Chest. Published online 2010. doi:10.1378/chest.09-1584

18. van den Ham HA, Klungel OH, Singer DE, Leufkens HGM, van Staa TP. Comparative performance of ATRIA, CHADS2, AND CHA2DS2-VASC RISK scores predicting stroke in patients with atrial fibrillation: results from a national primary care database. J Am Coll Cardiol. 2015;66(17):1851-1859. doi:10.1016/j.jacc.2015.08.033

19. Lip GYH, Frison L, Halperin JL, Lane DA. Identifying patients at high risk for stroke despite anticoagulation: a comparison of contemporary stroke risk stratification schemes in an anticoagulated atrial fibrillation cohort.Stroke. 2010;41(12):2731-2738. doi:10.1161/STROKEAHA.110.590257

20. Shen Mark J., Arora Rishi, Jalife José. Atrial myopathy. JACC: Basic to Translational Science. 2019;4(5):640-654. doi:10.1016/j.jacbts.2019.05.005

21. Rivner H, Mitrani RD, Goldberger JJ. Atrial myopathy underlying atrial fibrillation.Arrhythm Electrophysiol Rev. 2020;9(2):61-70. doi:10.15420/aer.2020.13

22. Ronsoni RM, Saffi MAL, Gonçalves MVM, Nakayama IH, Luz Leiria TL. A new vision at the interface of atrial fibrillation and stroke. Front Cardiovasc Med. 2021;8:689313. doi:10.3389/fcvm.2021.689313

23. Khurram IM, Dewire J, Mager M, et al. Relationship between left atrial appendage morphology and stroke in patients with atrial fibrillation. Heart Rhythm. 2013;10(12):1843-1849. doi:10.1016/j.hrthm.2013.09.065

24. Anselmino M, Scaglione M, Di Biase L, et al. Left atrial appendage morphology and silent cerebral ischemia in patients with atrial fibrillation. Heart Rhythm. 2014;11(1):2-7. doi:10.1016/j.hrthm.2013.10.020

25. He J, Tse G, Korantzopoulos P, et al. P-Wave indices and risk of ischemic stroke: a systematic review and meta-analysis. Stroke. 2017;48(8):2066-2072. doi:10.1161/STROKEAHA.117.017293

26. Huang Z, Zheng Z, Wu B, et al. Predictive value of $\mathrm{P}$ wave terminal force in lead V1 for atrial fibrillation: A meta-analysis. Ann Noninvasive Electrocardiol. 2020;25(4):e12739. doi:10.1111/anec.12739

27. Hsu PC, Lee WH, Chu CY, et al. Prognostic role of left atrial strain and its combination index with transmitral E-wave velocity in patients with atrial fibrillation.Sci Rep. 2016;6:17318. doi:10.1038/srep17318 
28. Leung M, van Rosendael PJ, Abou R, et al. Left atrial function to identify patients with atrial fibrillation at high risk of stroke: new insights from a large registry.Eur Heart J. 2018;39(16):1416-1425. doi:10.1093/eurheartj/ehx736

29. Tsao HM, Hu WC, Tsai PH, et al. The abundance of epicardial adipose tissue surrounding left atrium is associated with the occurrence of stroke in patients with atrial fibrillation. Medicine . 2016;95(14):e3260. doi:10.1097/MD.0000000000003260

30. Hijazi Z, Oldgren J, Andersson U, et al. Cardiac biomarkers are associated with an increased risk of stroke and death in patients with atrial fibrillation: a Randomized Evaluation of Long-term Anticoagulation Therapy (RE-LY) substudy. Circulation. 2012;125(13):1605-1616. doi:10.1161/CIRCULATIONAHA.111.038729

31. Berg DD, Ruff CT, Jarolim P, et al. Performance of the ABC Scores for Assessing the risk of stroke or systemic embolism and bleeding in patients with atrial fibrillation in ENGAGE AF-TIMI 48. Circulation. 2019;139(6):760-771. doi:10.1161/CIRCULATIONAHA.118.038312

32. Lee DC, Markl M, Ng J, et al. Three-dimensional left atrial blood flow characteristics in patients with atrial fibrillation assessed by 4D flow CMR. Eur Heart J Cardiovasc Imaging. 2016;17(11):1259-1268. doi:10.1093/ehjci/jev304

33. Markl M, Lee DC, Furiasse N, et al. Left atrial and left atrial appendage 4d blood flow dynamics in atrial fibrillation. Circ Cardiovasc Imaging. 2016;9(9):e004984. doi:10.1161/CIRCIMAGING.116.004984

34. Markl M, Lee DC, Ng J, Carr M, Carr J, Goldberger JJ. Left atrial 4-dimensional flow magnetic resonance imaging: stasis and velocity mapping in patients with atrial fibrillation. Invest Radiol. 2016;51(3):147-154. doi:10.1097/RLI.0000000000000219

35. Passman R. "Pill-in-Pocket" anticoagulation for atrial fibrillation: fiction, fact, or foolish? Circulation. 2021;143(23):2211-2213. doi:10.1161/CIRCULATIONAHA.121.053170 